\title{
A Study on Universal Factor Determining the Evolution of Surface Roughness During Electrochemical Polishing
}

Jianwei Ji ( $\boldsymbol{D}_{\text {jijw@sustech.edu.cn ) }}$

Southern University of Science and Technology

Khan M. Ajmal

Zejin Zhan

Rong Yi

Hui Deng

\section{Research Article}

Keywords: Electrochemical polishing, Tungsten, Surface roughness, Removed thickness, Viscous layer.

Posted Date: December 28th, 2021

DOI: https://doi.org/10.21203/rs.3.rs-1180131/v1

License: (c) (i) This work is licensed under a Creative Commons Attribution 4.0 International License.

Read Full License 


\title{
A study on universal factor determining the evolution of surface roughness during electrochemical polishing
}

\author{
Jianwei Ji ${ }^{\mathrm{a}}$, Khan M. Ajmal ${ }^{\mathrm{b}}$, Zejin Zhan ${ }^{\mathrm{b}}$, Rong Yi ${ }^{\mathrm{b}}$, Hui Deng ${ }^{\mathrm{b}, *}$ \\ a Academy for Advanced Interdisciplinary Studies, Southern University of Science and Technology, No. 1088, \\ Xueyuan Road, Shenzhen, Guangdong 518055, China \\ ${ }^{b}$ Department of Mechanical and Energy Engineering, Southern University of Science and Technology, No. 1088, \\ Xueyuan Road, Shenzhen, Guangdong 518055, China \\ *Corresponding author: dengh@ sustech.edu.cn (Hui Deng)
}

\section{Abstract}

Electrochemical polishing (ECP) is widely used for scratch- and damage-free finishing of metal components. Though the polishing effect of ECP has been confirmed in many researches, the influence of polishing parameters on evolution of surface roughness is still ambiguous owing to the use of different ECP systems. In this paper, the universal factor determining the evolution of surface roughness during ECP is studied by theoretical analysis as well as experiments. Theoretical analysis based on viscous layer mechanism demonstrates that the material removal thickness is the key parameter governing the roughness evolution of the polished surface regardless of other parameters including the voltage, current and electrolyte concentration and so forth. A series of experiments were designed and carried out to verify the proposed hypothesis. Both the experimental results and already published researches proved the validity and universality of the newly developed hypothesis on surface roughness evolution. This work is of great significance for further understanding the finishing mechanism of ECP and process control for its practical applications.

\section{Keywords}

Electrochemical polishing; Tungsten; Surface roughness; Removed thickness; Viscous layer. 


\section{Introduction}

The surface quality can seriously affect the appearance and working performance of metal components. Polishing is an important method to enhance the quality of a surface. Selecting an economical and efficient polishing method is crucial for the processing of high-quality surfaces. At present, chemical mechanical polishing (CMP) [1,2] is the most widely used polishing method. However, CMP is difficult for the finishing of intricate geometries, such as medical implants and optical molds. In addition, the polishing efficiency of CMP is seriously low which is unacceptable for mass production of metal components [3-5].

Besides, electrochemical polishing (ECP) [6-8] is a widely used metal finishing technique because of its high material removal efficiency, allowance for complex geometries, and no subsurface damage owing to its anodic dissolution mechanism. Since the discovery of ECP technology, relevant material removal mechanisms have been proposed and improved over time. According to the most widely accepted viscous layer theory [9], the dissolved products accumulate on the anode surface and form a highly resistive viscous layer during ECP. The viscous layer thickness determines its electrical resistance and the dissolution rate of the metal. Therefore, surface protrusions with shallow viscous layer are preferentially dissolved, and the rough surface becomes smooth. Later, passive film theory [10], duplex salt film model theory [11] and adsorbate acceptor mechanism [12] were proposed respectively. All these theories believed that the viscous layer is indispensable in the ECP process. Although these theories can explain the polishing phenomenon based on the material removal mechanism, they are ineffective to optimize the ECP parameters.

At present, numerous experiments are required to establish the evolution laws of surface roughness and optimize the polishing parameters of ECP. Some of the fundamental ECP 
parameters used for optimization are applied voltage/current, electrolyte concentration, electrolyte temperature, and polishing duration, which are also coupled simultaneously. In different researches on ECP, different systems are used by different researchers and it is almost impossible to fully control the amount parameters in the polishing process. Therefore, the literary-reported results are difficult to repeat and their conclusions provide extremely limited guidance about the process. And the reason is all the above conclusions are obtained based on specific polishing parameters. In summary, the current researches have not broken through the premise of experimental conditions or polishing parameters, and this means the governing parameter of the evolution of surface roughness in ECP is still undiscovered.

In this study, a novel understanding about the roughness evolution in ECP is proposed. Based on theoretical analysis and experimental validation, it is proved that the material removal thickness is the universal factor determining the evolution of surface roughness during ECP.

\section{Experimental}

Tungsten samples (Xin Ji Metal Materials Co., Ltd. China) used in experiments are more than $99.99 \%$ pure and taken from the same batch to ensure the uniformity of the samples. The size of the workpiece is $3 \times 13 \times 13 \mathrm{~mm}^{3}$. The samples were grounded for $30 \mathrm{~s}$ on a new 180 grit sandpaper. Later, the samples were further ground for another $10 \mathrm{~s}$ with 600 grit sandpaper. The roughness of the surface obtained by this process is about $200 \mathrm{~nm}$, which is nearly uniform throughout the workpiece surface. All the samples in this paper, unless otherwise specified, are prepared following the same surface treatment to obtain similar initial roughness and micro-morphology. 
Experiments were conducted in a $1000 \mathrm{ml}$ glass beaker containing the $\mathrm{NaOH}$ electrolyte. The workpiece was connected to the anode of the DC power supply. During polishing, only the front face $\left(13 \times 13 \mathrm{~mm}^{2}\right)$ of the workpiece was exposed to the electrolyte, while an insulating clamp covered the rest of the substrate. The cathode was a platinum sheet with an area of $20 \times 20 \mathrm{~mm}^{2}$. The polishing voltage was provided by the Keysight E3649A dual-channel DC power supply. The analytical grade $\mathrm{NaOH}$ electrolyte used in this study was provided by Merck, Germany.

The surface roughness before and after polishing was measured by white light interferometer (WLI, Taylor Hobson) with a 10X objective lens. The obtained image area was $1.66 \times 1.66 \mathrm{~mm}^{2}$, with a total of $2048 \times 2048$ pixels. The parameter of average surface roughness $\mathrm{Sa}$ (ISO25178) was used to evaluate surface quality. Besides, the power spectral density (PSD) function $[13,14]$ calculated by the Fourier transform of the discrete measured data was also used to analyze the roughness on specific spatial frequencies. A scanning electron microscope (SEM, Hitachi s-4800) was used to investigate the surface morphologies. The material removed after polishing was determined by a precision electronic balance (Mettler Toledo, ME203).

\section{ECP of tungsten}

The following experiment verified the performance of the polishing system. The polishing voltage used in the experiment was $15 \mathrm{~V}$, the electrolyte concentration was $1 \mathrm{wt} \%$, the interelectrode gap was $40 \mathrm{~mm}$, and the polishing time was $90 \mathrm{~min}$. Since the surface roughness largely depends on the selected measurement position. The repeated positioning analysis method was adopted to eliminate the error caused by the random measurement 
positions and accurately evaluate the polishing ability of ECP. The details about the repeated positioning method and the analysis of positioning accuracy can be found in Ref. [15]. The surface quality before and after ECP is shown in Fig. 1. Tungsten substrate images before and after polishing are shown in Fig. 1(a), where a dull surface is transformed into a mirror-like glossy surface after ECP. As shown by the SEM images in Fig. 1(b), there were noticeable grounding marks on the surface before ECP, which became smooth, and clear grain boundaries could be observed after ECP. The original surface roughness of $203 \mathrm{~nm}$ was optimized to $12.4 \mathrm{~nm}$ after $90 \mathrm{~min}$ of ECP, as shown in Fig. 1(c). Figure 1(d) shows the surface contours before and after polishing, which exclusively prove the polishing ability of the ECP.

Wang et al. [16] found that surface polishing strongly depends on the applied voltage. According to the results, surface polishing was realized when the polishing voltage was greater than $5 \mathrm{~V}$. However, the viscous layer did not develop, and the surface polishing was not realized below $5 \mathrm{~V}$; it was defined as the etching stage of ECP. The set of ECP parameters that can achieve surface polishing are defined as polishable parameters. Accordingly, it is essential to study the fundamental factors affecting the surface roughness evolution in the range of polishable parameters of ECP. Therefore, in Section 4, factors affecting the surface roughness evolution in ECP are theoretically analyzed based on the viscous layer theory. 


\section{(a)}

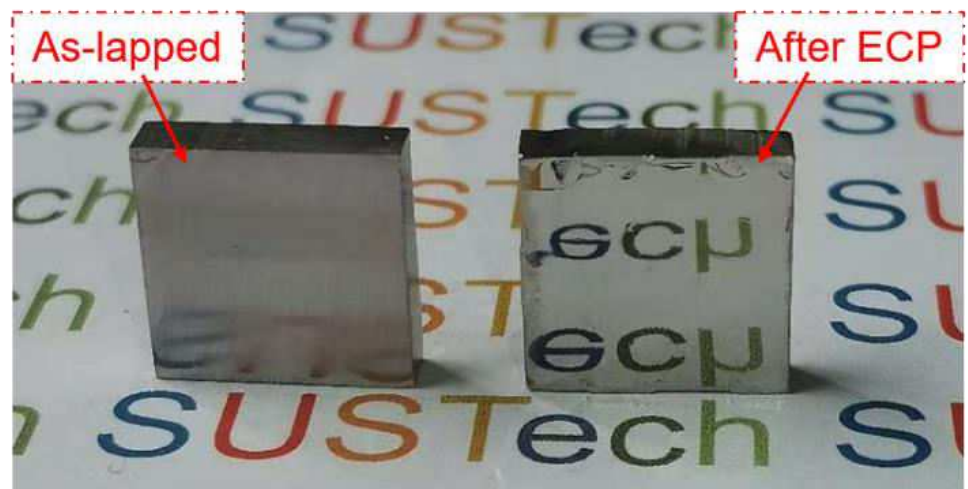

(b)

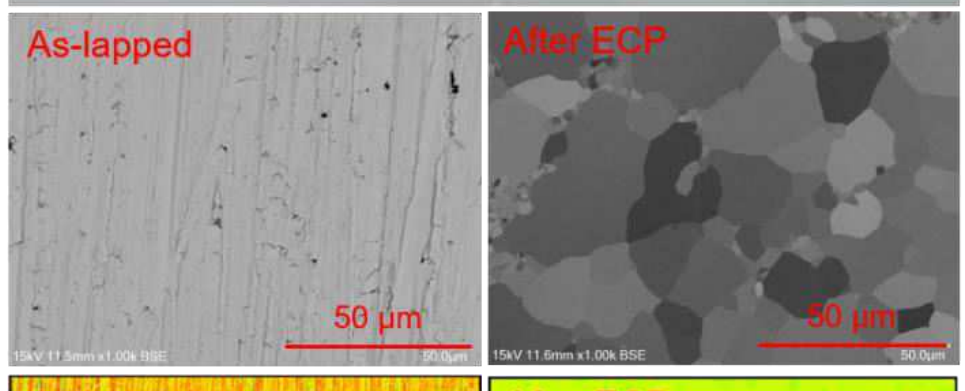

(c)
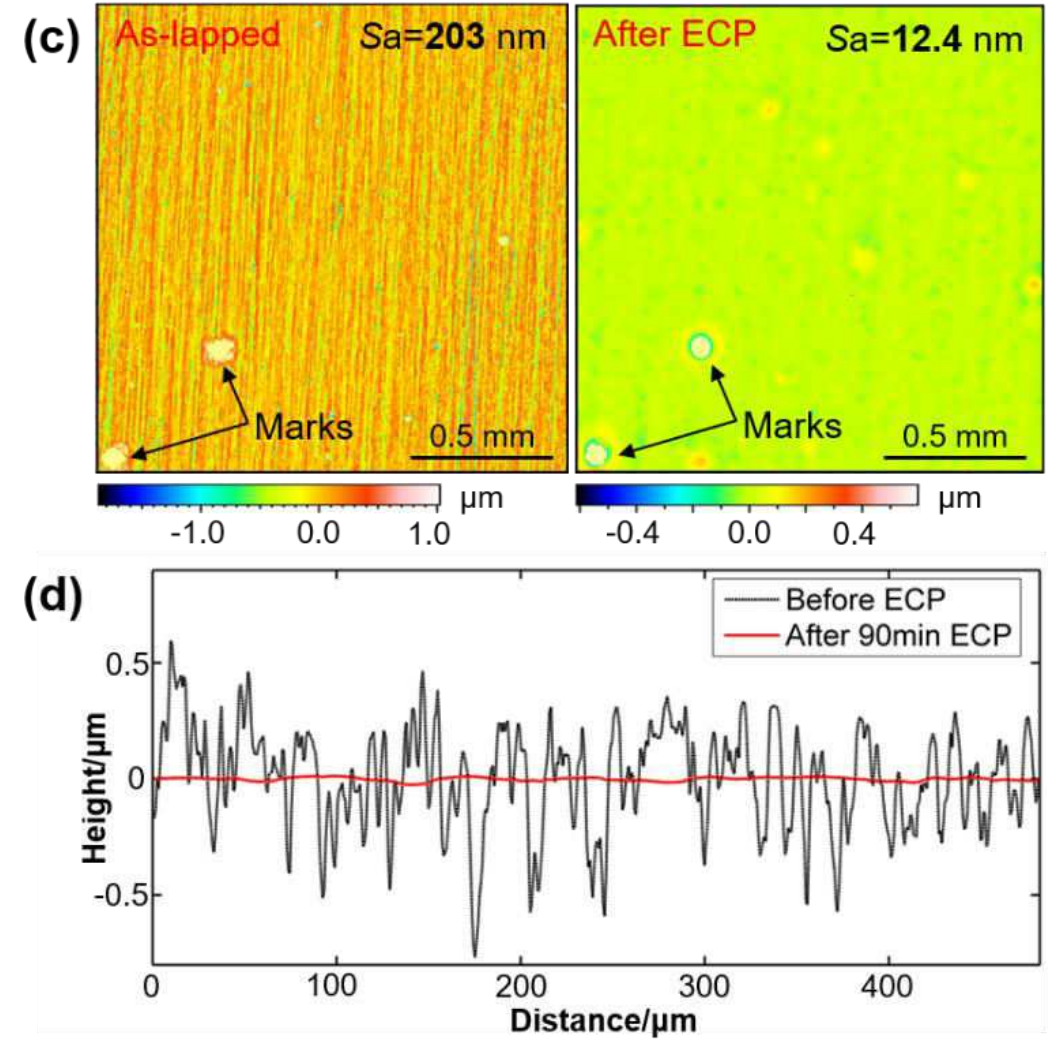

Fig. 1 Photos (a), SEM morphologies (b), WLI surface topographies (c), and surface contours (d) of Tungsten substrate before and after ECP

\section{Theoretical analysis}

The viscous layer theory is the most widely accepted electrochemical polishing mechanism, according to which a viscous layer with electrical resistance much higher than the electrolyte 
is formed on the anode surface during ECP. The schematic diagram of viscous layer theory is shown in Figure 2(a). The viscous layer at the convex position of the surface is relatively thinner and less resistive, allowing higher current flow and a higher material removal rate; on the contrary, the material removal rate at the concave position is lower. As the polishing progresses, the surface is gradually smoothed, and the surface roughness is reduced.
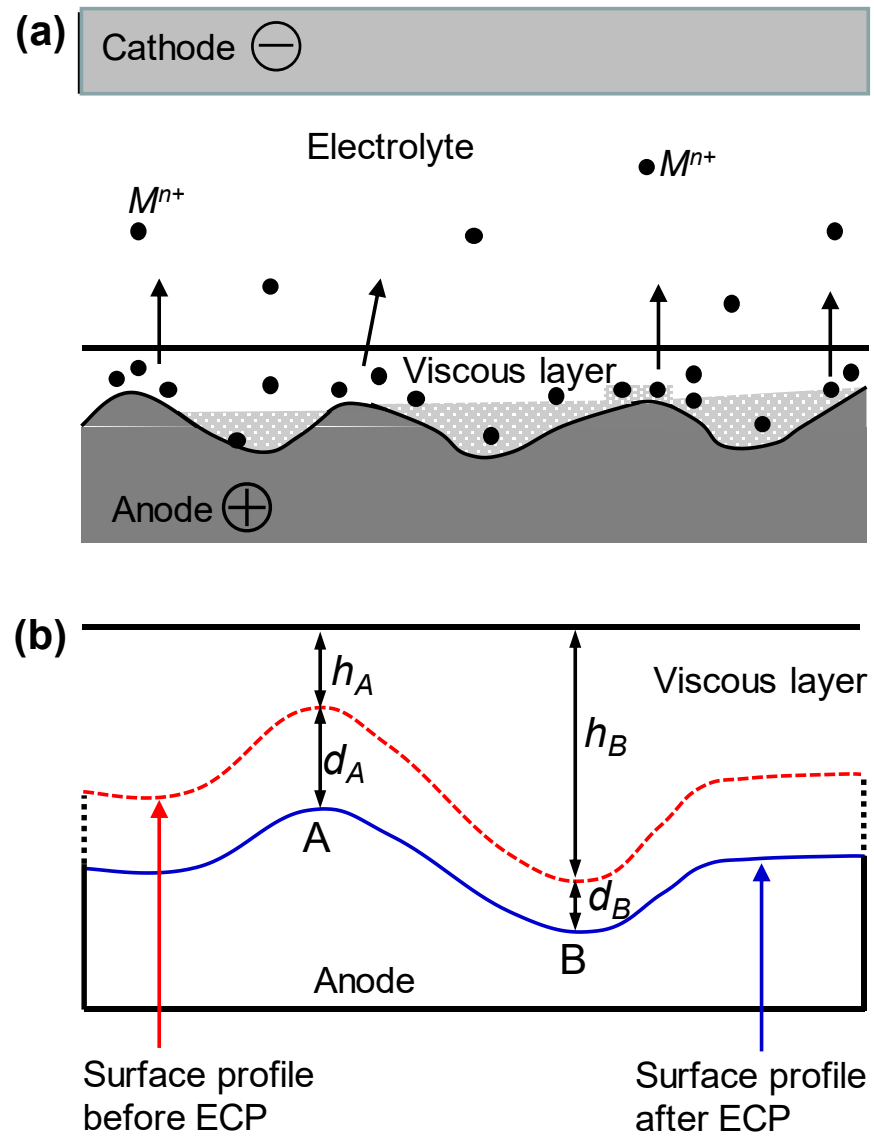

Fig. 2 (a) The schematic diagram and (b) simplified model of viscous layer theory

Figure 2(b) is a simplified model of viscous layer theory, where A and B are two arbitrary positions on the surface. Before polishing, the thickness of the viscous layer at position $\mathrm{A}$ is $h_{\mathrm{A}}$, and at position B is $h_{\mathrm{B}}$. After processing time $t$, the material removal thickness at position A is $d_{\mathrm{A}}$, and position $\mathrm{B}$ is $d_{\mathrm{B}}$.

The current flowing through point A can be expressed as follows: 


$$
I_{A}=\frac{U}{R_{A}}=\frac{U S}{k \cdot l_{A}}
$$

where $U$ is the applied voltage, $k$ is the resistivity of the viscous layer, $S$ is the unit area, and $l_{A}$ is the viscous layer thickness at position A.

It should be noted that when material removes from the surface, two possible changes may occur in the viscous layer: (i) the upper surface of the viscous layer remains unchanged and viscous layer becomes thicker, (ii) the upper surface of the viscous layer moves downward and the viscous layer remains unchanged. The first case is adopted in this paper mainly due to two reasons. As direct evidence, the thickness evolution of the viscous layer during ECP has been observed by using the in-situ X-ray nano-tomography, which shows an increase in the viscous layer thickness [17]. Secondly, current evolution (Fig. 3) during ECP also provides evidence of an increase in the viscous layer thickness. According to Fig. 3, the current continuously fluctuates during the ECP process. The gradually decreasing current means the viscous layer gradually thickens. A sudden fluctuation may be caused by the local breakdown or shedding of the viscous layer. An abrupt but minute change in current has little impact on the material removal process, therefore, in general, the viscous layer thickens during ECP.

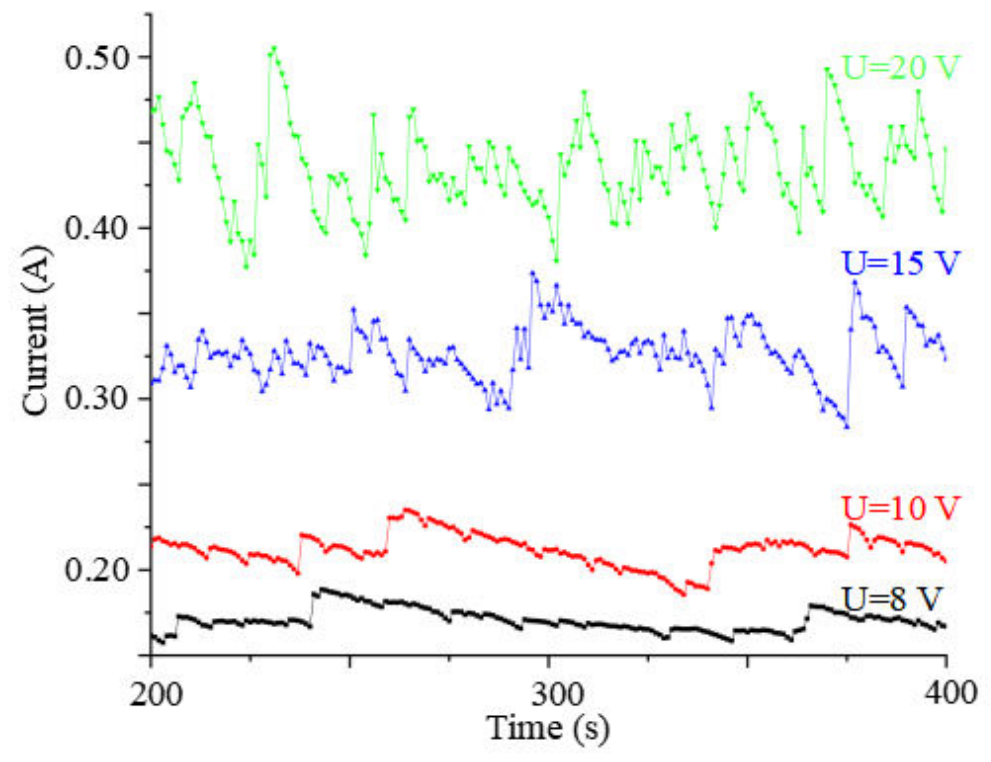

Fig. 3 Variation of current with time under different polishing voltage 
Based on the two pieces of evidence, we derived the formula following that the viscous layer $l_{A}$ thickens during ECP. For example, at the beginning, $l_{A}=h_{A}$ and changes to $l_{A}=h_{A}+d_{A}$ due to the removal of material thickness $d_{A}$ after processing time $t$. Therefore, the current $I_{A}$ also changes with the polishing.

The total charge $Q_{A}$ passing through position A in time $t$ can be expressed as follows:

$$
Q_{A}=I_{A} t=\int_{0}^{t} I_{A} d t=\int_{0}^{t} \frac{U S}{k \cdot\left(h_{A}+d_{A}\right)} d t
$$

During the polishing time $t$, the total removal volume at position A is as follows:

$$
V_{A}=\frac{m_{A}}{\rho_{W}}=\frac{\eta \cdot M_{W} \cdot Q_{A}}{q_{W} \cdot e \cdot N_{A} \cdot \rho_{W}}=\frac{\eta \cdot M_{W}}{q_{W} \cdot e \cdot N_{A} \cdot \rho_{W}} \int_{0}^{t} \frac{U S}{k \cdot\left(h_{A}+d_{A}\right)} d t=\frac{K \eta U S}{k} \int_{0}^{t} \frac{1}{h_{A}+d_{A}} d t
$$

Among them, $\eta$ is the current efficiency, $\eta Q_{A}$ is the charge required to remove tungsten atoms at position $\mathrm{A}, M_{W}$ is the relative atomic mass of tungsten, $q_{W}$ is the valence electron number of tungsten, $e$ is the charge of a single electron, $N_{\mathrm{A}}$ is the Avogadro constant, and $\rho_{W}$ is the density of tungsten metal. Since $M_{W}, q_{W}, e, \rho_{W}$, and $N_{\mathrm{A}}$ are all constants, let $K=\frac{M_{W}}{q_{W} \cdot e \cdot N A \cdot \rho_{W}}$, then $K$ is a constant and represents the material properties in the formula.

The material removal thickness $d_{A}$ during the polishing time $t$ at position $\mathrm{A}$ can be expressed as:

$$
d_{A}=\frac{V_{A}}{S}=\frac{K \eta U}{k} \int_{0}^{t} \frac{1}{h_{A}+d_{A}} d t
$$

Further solving equation (4), we get:

$$
d_{A}=\sqrt{2 \frac{K \eta U t}{k}+h_{A}^{2}}-h_{A}
$$

According to equation (5), the removal thickness positively correlates with voltage and processing time, which is consistent with the experimental results. The height difference 
between A and B positions after machining is given as follows:

$$
\Delta h^{\prime}=\left(h_{B}+d_{B}\right)-\left(h_{A}+d_{A}\right)=\sqrt{2 \cdot \frac{K \eta U t}{k}+h_{B}{ }^{2}}-\sqrt{2 \cdot \frac{K \eta U t}{k}+h_{A}{ }^{2}}
$$

According to equations (5) and (6), if the removal thickness $d_{A}$ at position $\mathrm{A}$ is the same in different processes, $\eta U t / k$ will be the same, then $\Delta h$ also remains the same. If $\mathrm{A}$ is the highest point on the surface, its height difference from a random point on the surface after polishing depends on the removal thickness $d$, which also determines the surface roughness after polishing.

Based on the above theoretical analysis, the ECP processed surface roughness is closely related to the removal thickness. However, the removal thickness itself is determined by ECP voltage, duration, and electrolyte concentration. According to the author's knowledge, this study is the first to propose variation in the surface roughness based on the material removal thickness. Previous studies have investigated surface roughness as a function of the polishing parameters, such as voltage and electrolyte concentration, while completely ignored the role of material removal thickness.

\section{Experimental validation}

A series of experiments were carried out to verify the previously discussed theoretical hypothesis. By adjusting the polishing duration, the same material removal thickness was achieved under different polishing parameters, and then the surface roughness after polishing was measured. In experiment set 1 , the effect of different voltages $(10 \mathrm{~V}, 15 \mathrm{~V}, 20 \mathrm{~V})$ was investigated under the same electrolyte concentration (1\%). In experiment set 2 , the effect of different electrolyte concentrations $(0.5 \%, 1 \%, 1.5 \%)$ was investigated under the same 
polishing voltage $(15 \mathrm{~V})$. The material removal thickness under different working conditions was calculated by combining the mass reduction with tungsten density and the workpiece geometry. The detailed experimental parameters and results are given in Table 1.

Table 1. Details of the experimental parameters and obtained results.

\begin{tabular}{lcccccc}
\hline & \multicolumn{3}{c}{ Experiment set 1 } & \multicolumn{3}{c}{ Experiment set 2 } \\
\hline Sample no. & $\mathrm{A}$ & $\mathrm{B}$ & $\mathrm{C}$ & $\mathrm{A}$ & $\mathrm{B}$ & $\mathrm{C}$ \\
Voltage/V & 10 & 15 & 20 & 15 & 15 & 15 \\
NaOH concentrations/wt\% & $1 \%$ & $1 \%$ & $1 \%$ & $0.5 \%$ & $1 \%$ & $1.5 \%$ \\
Polishing time/min & 34 & 20 & 13 & 76 & 23 & 16 \\
Mass reduction/g & 0.055 & 0.056 & 0.056 & 0.063 & 0.066 & 0.063 \\
Removal thickness/ $\mu \mathrm{m}$ & 16.819 & 17.125 & 17.125 & 19.266 & 20.183 & 19.266 \\
\hline
\end{tabular}

Figure 4 shows the PSD curves and WLI topographies obtained after experiment set 1 (see Fig. 4(a)) and experiment set 2 (see Fig. 4(b)). It should be noted that the PSD curves before polishing are coincident, which describe similar features of the substrate obtained after initial grounding. After both sets of polishing experiments, the PSD curves decreased significantly due to the reduction in surface roughness. Besides, PSD curves after polishing are also coincident, which is attributed to the similar surface roughness. The WLI topographies of workpieces after polishing also endorsed the PSD results. In conclusion, if the initial surfaces are identical, comparable final surface roughness values can be achieved by removing the same thickness regardless of the applied voltage and electrolyte concentration. 

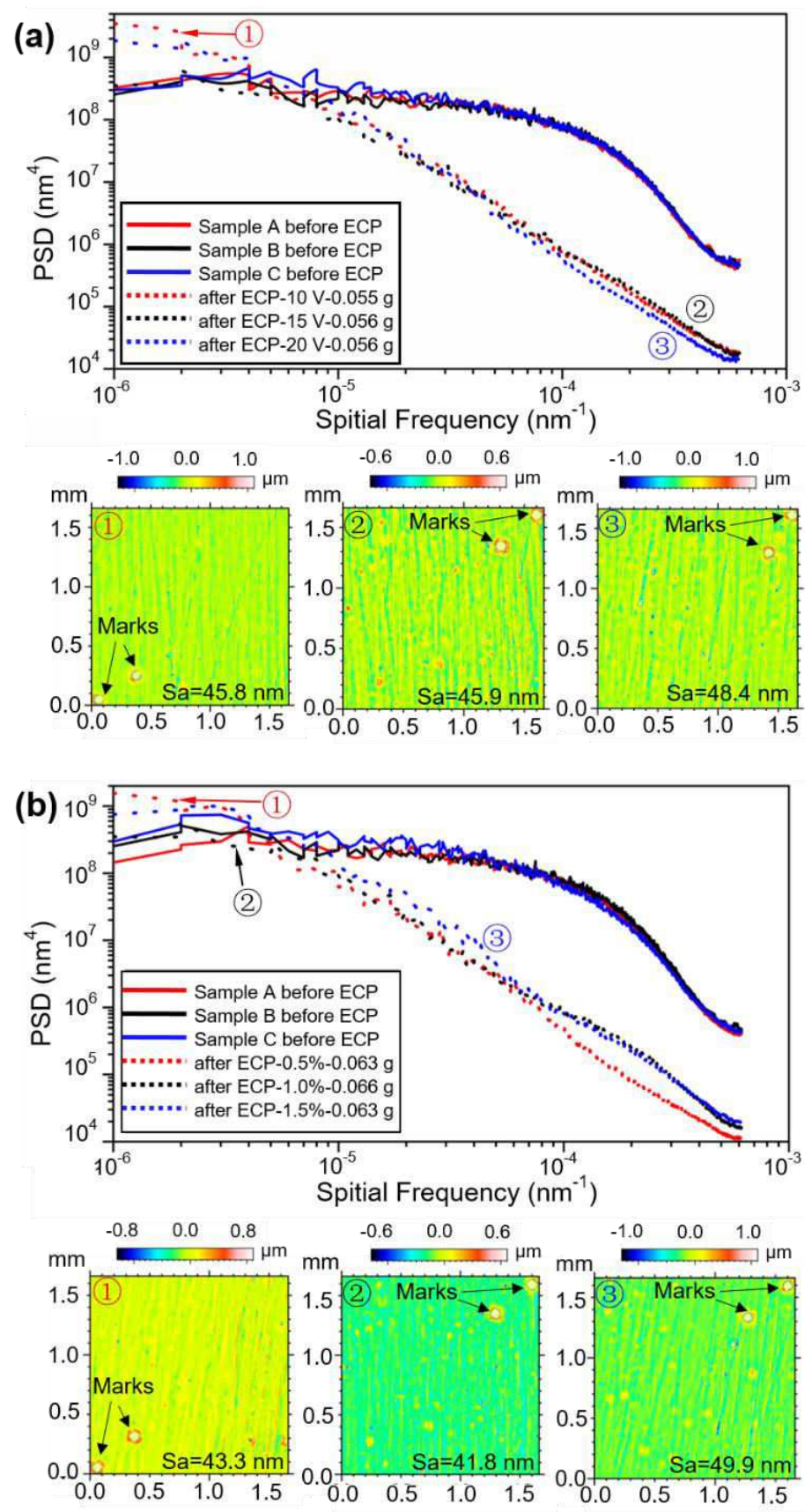

Fig. 4 The PSD curves and the WLI topographies of the workpieces in (a) experimental set 1 and (b) experimental set 2

Following the procedure outlined in Section 2, a series of surfaces with similar roughness $(\sim 200 \mathrm{~nm})$ were prepared. All the workpieces were marked to specify the surface measurement position before and after polishing. Subsequently, the workpieces were polished for the different durations by randomly selecting applied voltage and electrolyte concentrations while keeping the rest of the parameters fixed. According to the results shown 
in Fig. 5, similar removal thickness on the abscissa finds no significant difference in the surface roughness. It means, as long as the material removal thickness is identical, post-processing surface roughness remains similar regardless of the polishing parameters.

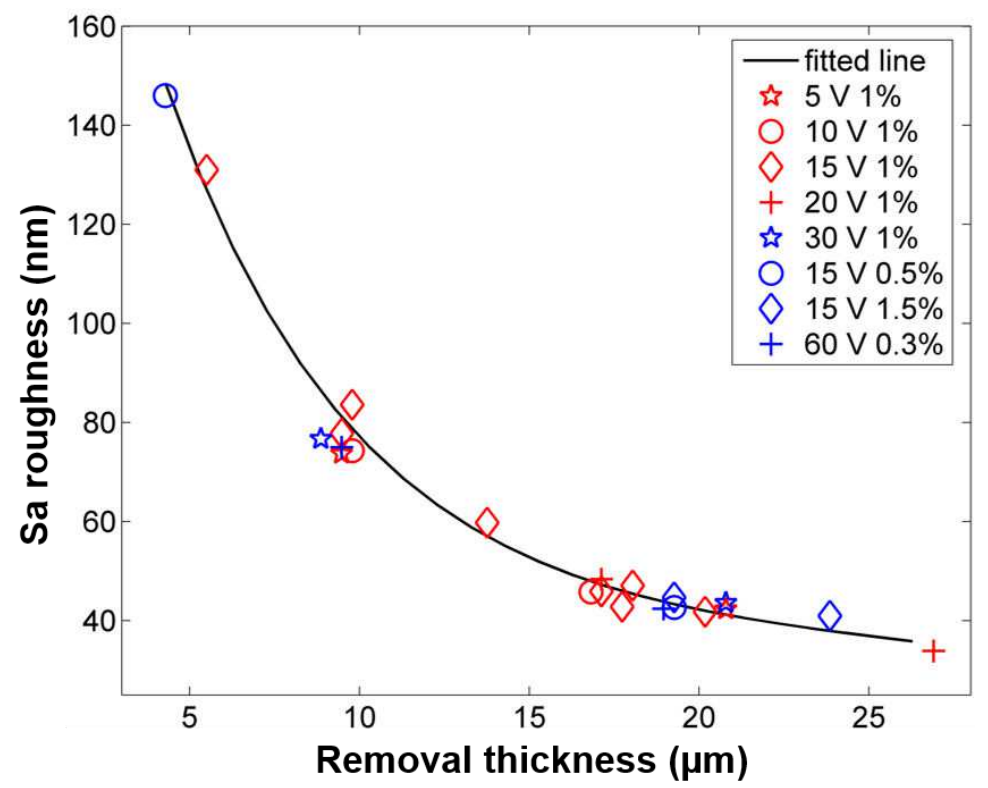

Fig. 5 Evolution of surface roughness with material removal thickness in ECP technology

The proposed conclusion is further verified by the ECP experiments on stainless steel and copper, the most widely used industrial metals. The electrolyte used for polishing stainless steel is phosphoric acid and sulfuric acid with a volume ratio of 2:1, and the electrolyte used for polishing copper is phosphoric acid and deionized water with a volume ratio of $4: 1$. The initial surface roughness of all the samples was $\sim 200 \mathrm{~nm}$. Figure 6 shows the surface roughness of stainless steel (Figs. 6(a-c)) and copper (Figs. 6(d-f)) after ECP at different applied currents $(1,2$, and 3 A) while keeping the material removal thickness constant. As evident from Fig. 6, the surface roughness values for each material are comparative after ECP when the material removal thickness is similar. In addition, it can be stated that the proposed mechanism is independent of the applied current and nature of electrolytes when material removal thickness is constant, which is also in agreement with the theoretical analysis given in Section 4. 

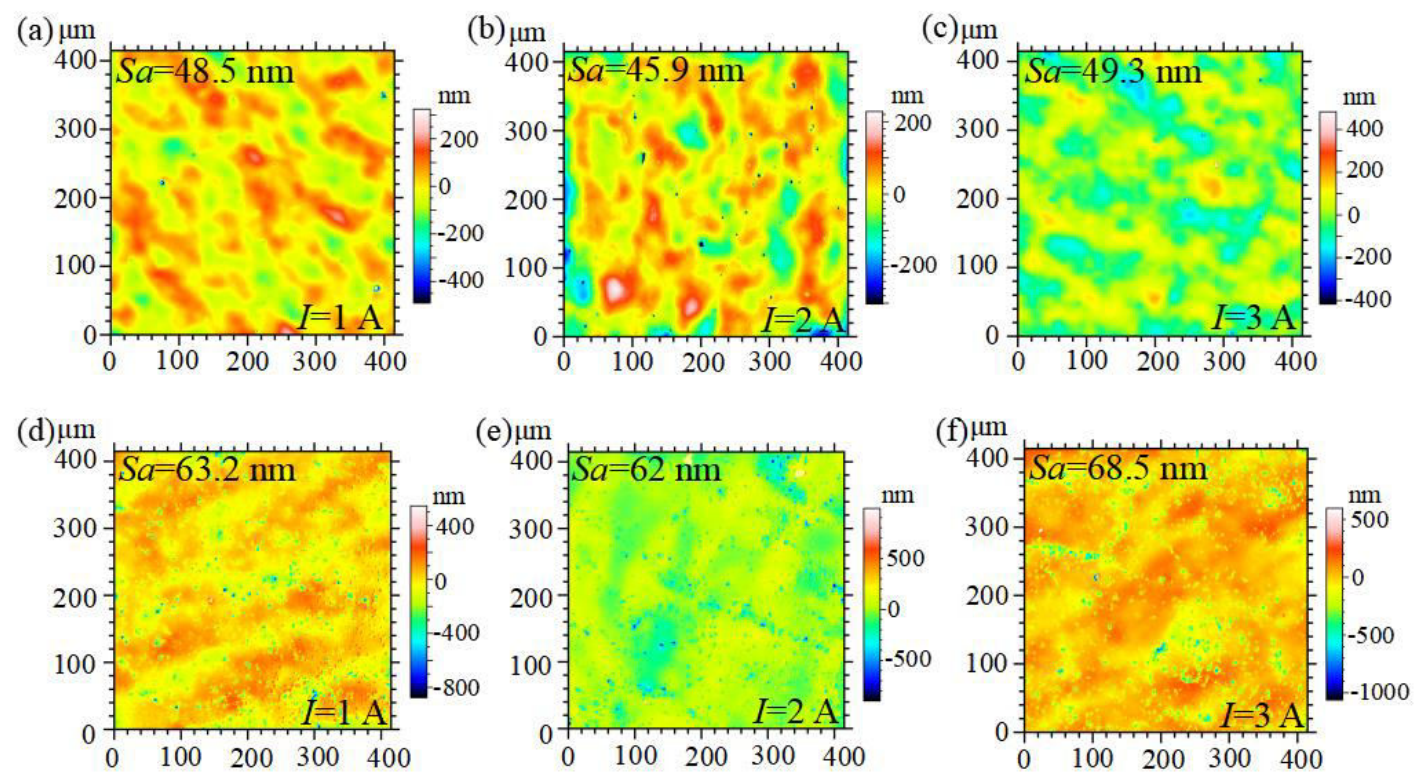

Fig. 6 Results of stainless steel (a)(b)(c) and copper (d)(e)(f) after ECP with different current

In addition, these findings are in good agreement with the already published literature. For example, Simka et al. [18] processed Ti-13Nb-13Zr alloy in ammonium fluoride and sulfuric acid electrolyte and observed a slight change in the surface roughness with an increase in the current density (40 to $60 \mathrm{~A} \cdot \mathrm{dm}^{-2}$ ) at a fixed electric charge, shown as Fig. 7(a). However, a fundamental in-depth analysis was not performed. Similarly, stainless steel 316L was processed in phosphoric acid and sulphuric acid electrolyte by using the Taguchi method [19]. According to the results shown in Fig. 7(b), under the same electrode gap, the polished surface roughness is comparable when the product of applied current and machining time is constant. In addition, the higher the value $Q$, the better the roughness, that means the roughness is not determined by the voltage or current, but by the material removal thickness. Such results are in good agreement with formula derivation in Section 4. Compared with the experimental results and the results reported by literature, it is easy to conclude that the hypothesis we proposed is generic, regardless of the polished material or working electrolyte. Furthermore, these results agree with the theoretical analysis since the parameter $K$ representing material properties is like a coefficient. Regardless of the changes in the value of 
$K$, the conclusion remains valid.
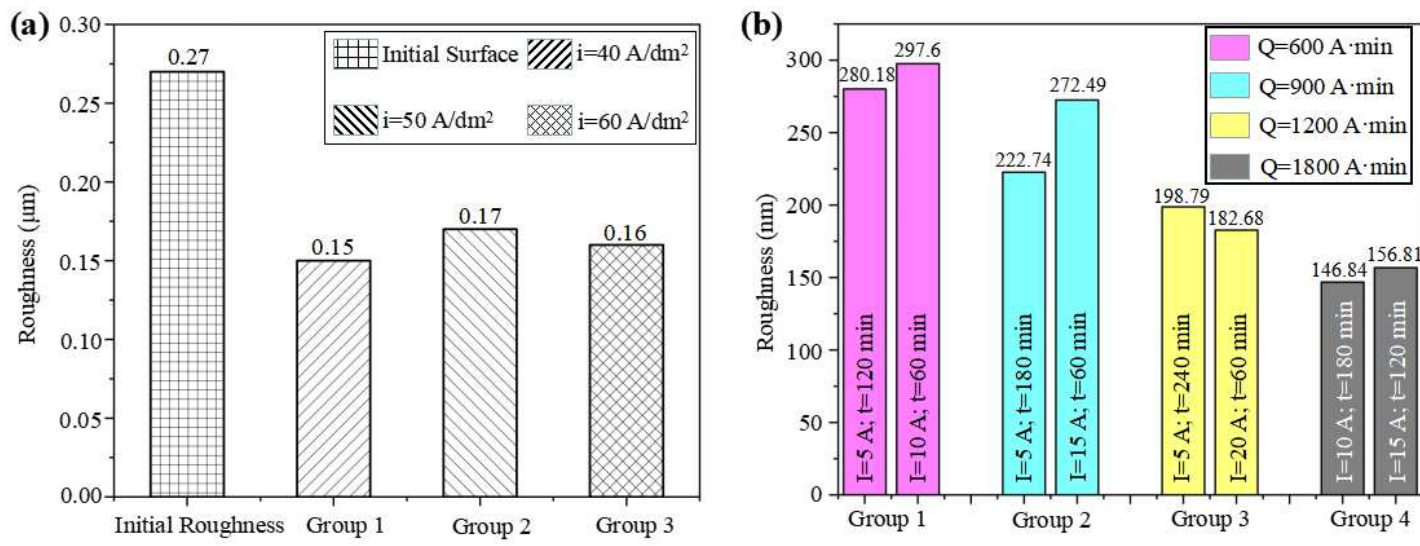

Fig. 7 Experimental results in the published literature (a) literature 17, (b) literature 18

It should be pointed out that the preceding conclusion of surface roughness dependence on the material removal thickness regardless of polishing parameters is only valid in the polishing stage of ECP. For example, if the voltage is too low and belongs to the so-called etching stage or the electrolyte concentration is inappropriate, surface polishing cannot be achieved regardless of the polishing duration and the material removal thickness.

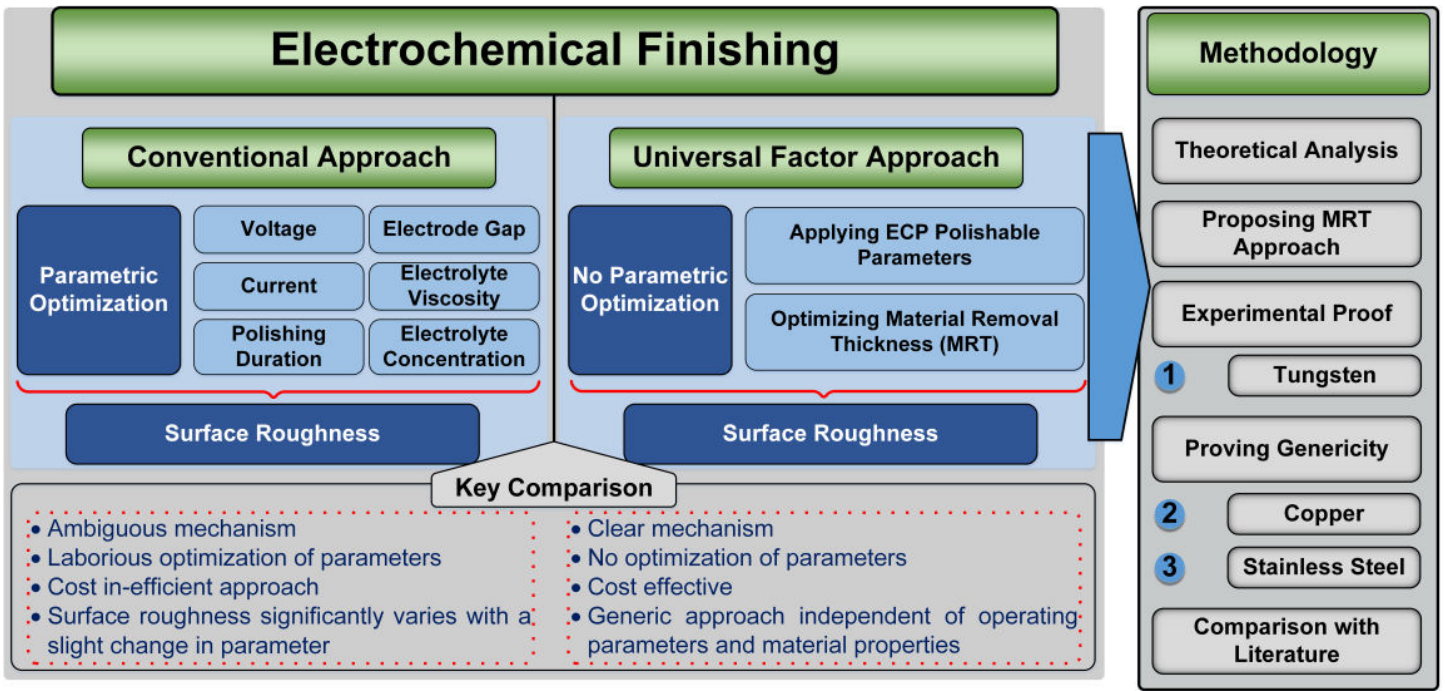

Fig. 8 A summary of the research methodology and its significance of the universal factor approach

A key comparison have been presented between the conventional approach and the universal factor approach in Fig. 8. Since the discovery of ECP technology, much research 
work on the surface roughness evolution during ECP has been conducted. Conventional approach usually obtained the evolution laws of surface roughness at the premise of specific experimental conditions or polishing parameters, which leads to extremely limited guidance for the practical application of ECP. In contrast, our work reveals the decisive factors affecting surface roughness evolution during ECP and obtains the essential evolution law of surface roughness independent of polishing parameters. The theoretical derivation, experimental results, and results reported by researchers all strongly support the correctness and universality of these conclusions.

\section{Conclusions}

Due to the material removal nature of anodic dissolution, ECP has many advantages and is a promising finishing technology, especially for metals with high hardness. Based on the fundamental theory of ECP, this paper theoretically investigates the influence of polishing parameters on the surface roughness during the polishing process and proposes a new hypothesis that the polished surface roughness is mainly influenced by the removal thickness regardless of voltage, electrolyte concentration and other parameters. The validity and universality of the newly developed hypothesis are further verified through experiments and supported by the published literature.

\section{Ethical Approval}

Not applicable 


\section{Consent to Participate}

Not applicable

\section{Consent to Publish}

Not applicable

\section{Availability of data and materials}

All data generated or analyzed during this study are included in this published article.

\section{Declaration of Competing Interest}

The authors declare that there are no conflicts of interest.

\section{Acknowledgments}

This work is financially supported by the National Natural Science Foundation of China (Grant No. 52035009, 52005243) and the research fund from the Science and Technology Innovation Committee of Shenzhen Municipality (JCYJ20200109141003910), Shenzhen, China. The authors acknowledge the assistance of SUSTech Core Research Facilities. 


\section{References}

1. H. Deng, K. Endo, K. Yamamura (2015) Competition between surface modification and abrasive polishing: a method of controlling the surface atomic structure of $4 \mathrm{H}-\mathrm{SiC}$ (0001). Sci Rep 5: 8947. https://doi.org/10.1038/srep08947.

2. L. Zhou, V. Audurier, P. Pirouz, J. Powell (1997) Chemomechanical polishing of silicon carbide. J Electrochem Soc 144:161-163. https://doi:10.1149/1.1837711.

3. W. Zhai, B. Gao, J. Chang, H. Wang (2019) Optimization of Ultrasonic-Assisted Polishing SiC Through CFD Simulation. Nanomanuf Metrol 2:36-44. https://doi.org/10.1007/s41871-018-0033-8.

4. J. Murata, K. Yodogawa, K. Ban (2017) Polishing-pad-free electrochemical mechanical polishing of single-crystalline $\mathrm{SiC}$ surfaces using polyurethane- $\mathrm{CeO}_{2}$ core-shell particles. Int $\mathrm{J}$ Mach Tool Manuf 114:1-7. https://doi.org/10.1016/j.ijmachtools.2016.11.007.

5. H. Deng, K. Hosoya, Y. Imanishi, K. Endo, K. Yamamura (2015) Electro-chemical mechanical polishing of single-crystal $\mathrm{SiC}$ using $\mathrm{CeO}_{2}$ slurry. Electrochem Commun 52:5-8. https://doi.org/10.1016/j.elecom.2015.01.002.

6. H. Deng, R. Huang, K. Liu, X.Q. Zhang (2017) Abrasive-free polishing of tungsten alloy using electrochemical etching. Electrochem Commun 82:80-84. https://doi.org/10.1016/j.elecom.2017.07.030.

7. W. Han, F.Z. Fang (2019) Fundamental aspects and recent developments in electropolishing. Int $J$ Mach Tool Manuf 139:1-23. https://doi.org/10.1016/j.ijmachtools.2019.01.001.

8. K.M. Ajmal, R. Yi, Z.J. Zh, J.W. Ji, X.Q. Zhang, H. Deng (2021) Highly efficient smoothing of Inconel 718 via electrochemical-based isotropic etching polishing. Precis Eng 71:119-129. https://doi.org/10.1016/j.precisioneng.2021.03.005.

9. H.F. Walton (1936) The Anode Layer in the Electrolytic Polishing of Copper. J Electrochem Soc 97:219-226. https://doi.org/10.1149/1.2777996.

10.T.P. Hoar, J.A.S. Mowat (1950) Mechanism of electropolishing. Nature 165:64-65. https://doi.org/10.1038/165064a0.

11.R.D. Grimm, A.C. West, D. Landolt (1992) AC impedance study of anodically formed salt films on iron in chloride solution. $J$ Electrochem Soc 139:1622-1629. https://doi.org/10.1149/1.2069467.

12.M. Matlosz, S. Magaino, D. Landolt (1994) Impedance analysis of a model mechanism for acceptor-limited electropolishing. J Electrochem Soc 141:410-418. https://doi.org/10.1149/1.2054741.

13.N.I. Chkhalo, S.A. Churin, A.E. Pestov, N.N. Salashchenko, Y.A. Vainer, M.V. Zorina (2014) Roughness measurement and ion-beam polishing of super-smooth optical surfaces of fused quartz and optical ceramics. Opt Express 22:20094-20106. https://doi.org/10.1364/OE.22.020094. 
14.J.D. Hoyo, H. Choi, J.H. Burge, G.H. Kim, D.W. Kim (2017) Experimental power spectral density analysis for mid- to highspatial frequency surface error control. Appl Opt 56:5258-5267. https://doi.org/10.1364/AO.56.005258.

15.J.W. Ji, W. Fan, W. Gao, C. Wang, Y.F. Zhang, M. Xu, F. Ji (2019) Investigation of Roughness Evolution of Ion Sputtered Fused Silica Surface. Appl Opt 58:5388-5396. https://doi.org/10.1364/AO.58.005388.

16.F. Wang, X. Zhang, H. Deng (2019) A comprehensive study on electrochemical polishing of tungsten. Appl Surf Sci 475:587-597. https://doi.org/10.1016/j.apsusc.2019.01.020.

17.Maryana I. Nave, Jason P. Allen, Yu-chen Karen Chen-Wiegart, Jun Wang, Surya R. Kalidindi, Konstantin G. Kornev (2015) In situ X-ray nanotomography of metal surfaces during electropolishing. Scientific Reports 5:15257. https://doi.org/10.1038/srep15257.

18.W. Simka, M. Mosiałk, G. Nawrat, P. Nowak, J. Żak, J. Szade, A. Winiarski, A. Maciej, L.S. Warszynska (2012) Electrochemical polishing of Ti-13Nb-13Zr alloy. Surf Coat Tech 213:239-246. https://doi.org/10.1016/j.surfcoat.2012.10.055.

19.S.G. Choi, S.H. Kim, W.K. Choi, E.S. Lee (2016) The optimum condition selection of electrochemical polishing and surface analysis of the stainless steel 316L by the Taguchi method. Int J Adv Manuf Technol 82:1933-1939. https://doi.org/10.1007/s00170-015-7404-8. 